\title{
Quantifying spin Hall angles from spin pumping: Experiments and Theory
}

\author{
O. Mosendz, ${ }^{1, \text { * }}$ J. E. Pearson, ${ }^{1}$ F. Y. Fradin, ${ }^{1}$ G. E. W. Bauer, ${ }^{2}$ S. D. Bader,${ }^{1,3}$ and A. Hoffmann ${ }^{1,3}$ \\ ${ }^{1}$ Materials Science Division, Argonne National Laboratory, Argonne, IL 60439, USA \\ ${ }^{2}$ Kavli Institute of NanoScience, Delft University of Technology, 2628 CJ Delft, The Netherlands \\ ${ }^{3}$ Center for Nanoscale Materials, Argonne National Laboratory, Argonne, IL 60439, USA
}

(Dated: May 21, 2018)

\begin{abstract}
Spin Hall effects intermix spin and charge currents even in nonmagnetic materials and, therefore, ultimately may allow the use of spin transport without the need for ferromagnets. We show how spin Hall effects can be quantified by integrating $\mathrm{Ni}_{80} \mathrm{Fe}_{20} \mid$ normal metal $(\mathrm{N}$ ) bilayers into a coplanar waveguide. A $d c$ spin current in $\mathrm{N}$ can be generated by spin pumping in a controllable way by ferromagnetic resonance. The transverse $d c$ voltage detected along the $\mathrm{Ni}_{80} \mathrm{Fe}_{20} \mid \mathrm{N}$ has contributions from both the anisotropic magnetoresistance (AMR) and the spin Hall effect, which can be distinguished by their symmetries. We developed a theory that accounts for both. In this way, we determine the spin Hall angle quantitatively for $\mathrm{Pt}, \mathrm{Au}$ and Mo. This approach can readily be adapted to any conducting material with even very small spin Hall angles.

PACS numbers: 72.25.Rb, 75.47.-m, 76.50.+g
\end{abstract}

The spin-orbit interaction gives rise to spin-dependent scattering that can couple charge and spin currents in conducting materials. Resultant spin Hall effects [1-3] may therefore display spin-dependent transport even in materials and device structures that do not contain ferromagnetic materials. The effectiveness of this spin-charge conversion can be quantified by the material-specific spin Hall angle $\gamma$, which is given by the ratio of spin Hall and charge conductivities [4] and can be quantified by magnetotransport measurements [5 - 9 . However, $\gamma$ values reported in the literature vary over several orders of magnitude even for nominally identical materials (i.e., Au: $\gamma<0.022$ [10] and $\gamma=0.113$ [8], and Pt: $\gamma=0.0037$ [7] and $\gamma=0.08$ [11]). In order to better understand spin-dependent scattering and its potential use for spintransport applications it is therefore highly desirable to find a robust method to quantify $\gamma$. Here we demonstrate an approach whose sensitivity can be adjusted to measure even very small values of $\gamma$.

Previous work on ferromagnetic resonance (FMR) in magnetic multilayers has shown that spin pumping can create pure spin currents in normal metals (N) 12 14]. Upon excitation of FMR, the time-varying magnetization inside the ferromagnet $(\mathrm{F})$ generates an instantaneous spin current $j_{s}$ at the $\mathrm{F} \mid \mathrm{N}$ interface given by [15, 16]:

$$
j_{s} \overrightarrow{\mathrm{s}}=\frac{\hbar}{8 \pi} \operatorname{Re}\left(2 g_{\uparrow \downarrow}\right)\left[\overrightarrow{\mathrm{m}} \times \frac{\partial \overrightarrow{\mathrm{m}}}{\partial t}\right],
$$

where $\overrightarrow{\mathrm{m}}$ is the unit vector of the magnetization, $\overrightarrow{\mathrm{s}}$ is the unit vector of the spin current polarization, and $\operatorname{Re}\left(g_{\uparrow \downarrow}\right)$ is the real part of the spin mixing conductance. Under a simple precession [17] the spin pumping induces a net $d c$ spin current and by time-averaging Eq. (11) we get:

$$
j_{s, d c}^{0}=\frac{\hbar \omega}{4 \pi} R e g_{\uparrow \downarrow} \sin ^{2} \theta,
$$

where $\omega$ is the driving frequency and $\theta$ is the cone angle of the precession of $\vec{m}$. This spin current decays due to spin relaxation and diffusion in $\mathrm{N}$, such that the spin current at distance $z$ from the interface is:

$$
j_{s, d c}(z)=j_{s, d c}^{0} \frac{\sinh \left(\left(z-t_{N}\right) / \lambda_{s d}\right)}{\sinh \left(t_{N} / \lambda_{s d}\right)}
$$

where $\lambda_{s d}$ is the materials specific spin diffusion length and $t_{N}$ is the thickness of the $\mathrm{N}$ layer.

The spin current gives rise to a transverse charge current $\vec{j}_{c}^{I S H}(z)=\gamma(2 e / \hbar)\left[\vec{j}_{s, d c}(z) \times \vec{\sigma}\right]$ due to the inverse spin Hall effect (ISHE). It has already been demonstrated that this transverse charge current can be observed as a $d c$ voltage $18-20]$. Here we show how an approach based on spin pumping can be applied to various $\mathrm{F} \mid \mathrm{N}$ combinations. We identify two contributions to the $d c$ voltage that stem from anisotropic magnetoresistance (AMR) and spin Hall effect, respectively, and can be distinguished by their symmetries. Furthermore, we present a self-consistent theory that enables quantification of the spin Hall angle with high accuracy.

We integrated $\mathrm{F} \mid \mathrm{N}$ bilayers into coplanar waveguides with additional leads for measuring a $d c$ voltage along the sample. This is shown in Fig. 目 for a $\mathrm{Ni}_{80} \mathrm{Fe}_{20}(\mathrm{Py}) \mid \mathrm{Pt}$ bilayer, with lateral dimensions of $2.92 \mathrm{~mm} \times 20 \mu \mathrm{m}$ and 15 -nm thick individual layers. The bilayer was prepared by optical lithography, sputter deposition, and lift-off on a GaAs substrate. Subsequently we prepared Ag contacts for the voltage measurements, covered the whole structure with $100-\mathrm{nm}$ thick $\mathrm{MgO}$ (for $d c$ insulation between bilayer and waveguide), and defined a $30-\mu \mathrm{m}$ wide and 200-nm thick Au coplanar waveguide on top of the bilayer. Similar samples were prepared with $60-n m$ thick $\mathrm{Au}$ and Mo layers replacing Pt.

The FMR was excited by a $4-\mathrm{GHz}, 100-\mathrm{mW}$ rf excitation, while applying a $d c$ magnetic field $\vec{H}_{d c}$ at $\alpha=45^{\circ}$ with respect to the waveguide [see Fig. 1(c)]. The FMR signal was determined from the impedance of the waveguide 21]; simultaneously the $d c$ voltage was measured as 


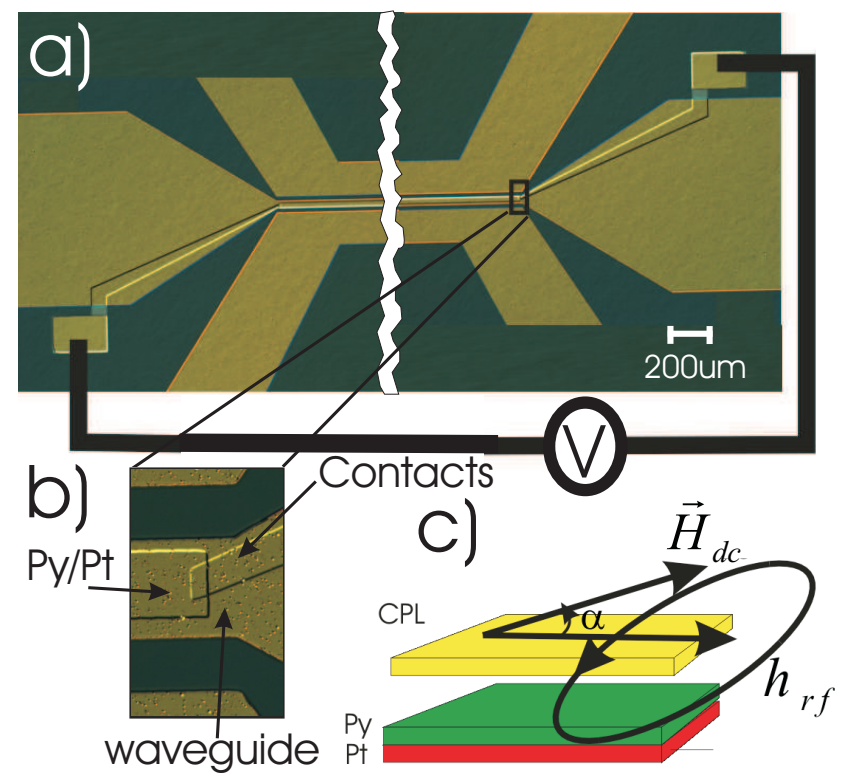

FIG. 1: (Color online) Experimental setup. (a) Optical image of the $\mathrm{Py} \mid \mathrm{Pt}$ bilayer integrated into the coplanar waveguide. (b) Voltage contacts added at the end of the bilayer for measuring the voltage along the direction of the waveguide. (c) Directions of the applied $d c$ magnetic field $\vec{H}_{d c}$ and the $r f$ driving field $\vec{h}_{r f}$ with respect to the bilayer and waveguide.

a function of $\vec{H}_{d c}$. Figure 2 shows this for a Py|Pt bilayer and a Py single layer, where both FMR peak positions are similar and consistent with the Kittel formula:

$$
\left(\omega / \gamma_{g}\right)^{2}=H_{d c}\left(H_{d c}+4 \pi M_{s}\right),
$$

where $\gamma_{g}=g e / 2 m c$ is the gyromagnetic ratio, $g$ is the electron g-factor and $M_{s}=852 \mathrm{G}$ is the saturation magnetization for Py. The FMR linewidths (HWHM) extracted from fits to Lorentzian absorption functions are $\Delta H_{P t / P y}=16.9$ Oe for Py $\mid \mathrm{Pt}$ and $\Delta H_{P y}=12.9$ Oe for Py. The difference in FMR linewidth can be attributed to the loss of spin momentum in Py due to relaxation of the spin accumulation in Pt. This permits the determination of the additional interface damping due to spin pumping [22], which in turn provides the interfacial spin mixing conductance as:

$$
g_{\uparrow \downarrow}=\frac{4 \pi \gamma_{g} M_{s} d_{P y}}{g \mu_{B} \omega}\left(\Delta H_{P t / P y}-\Delta H_{P y}\right),
$$

where $d_{P y}$ is the Py layer thickness and $\mu_{B}$ is the Bohr magneton (spin backflow being disregarded since Pt is an efficient spin sink). The calculated value for $g_{\uparrow \downarrow}=$ $2.1 \times 10^{19} \mathrm{~m}^{-2}$ is somewhat smaller than the previously reported $2.58 \times 10^{19} \mathrm{~m}^{-2}$ [23, 24], but Cao et al. 25] showed that for high power $r f$ excitation, the spin mixing conductance is reduced due to the loss of coherent spin precession in the ferromagnet.

Figure 2(c) shows the $d c$ voltage measured along the samples. For the $\mathrm{Py} \mid \mathrm{Pt}$ sample we observe a resonant

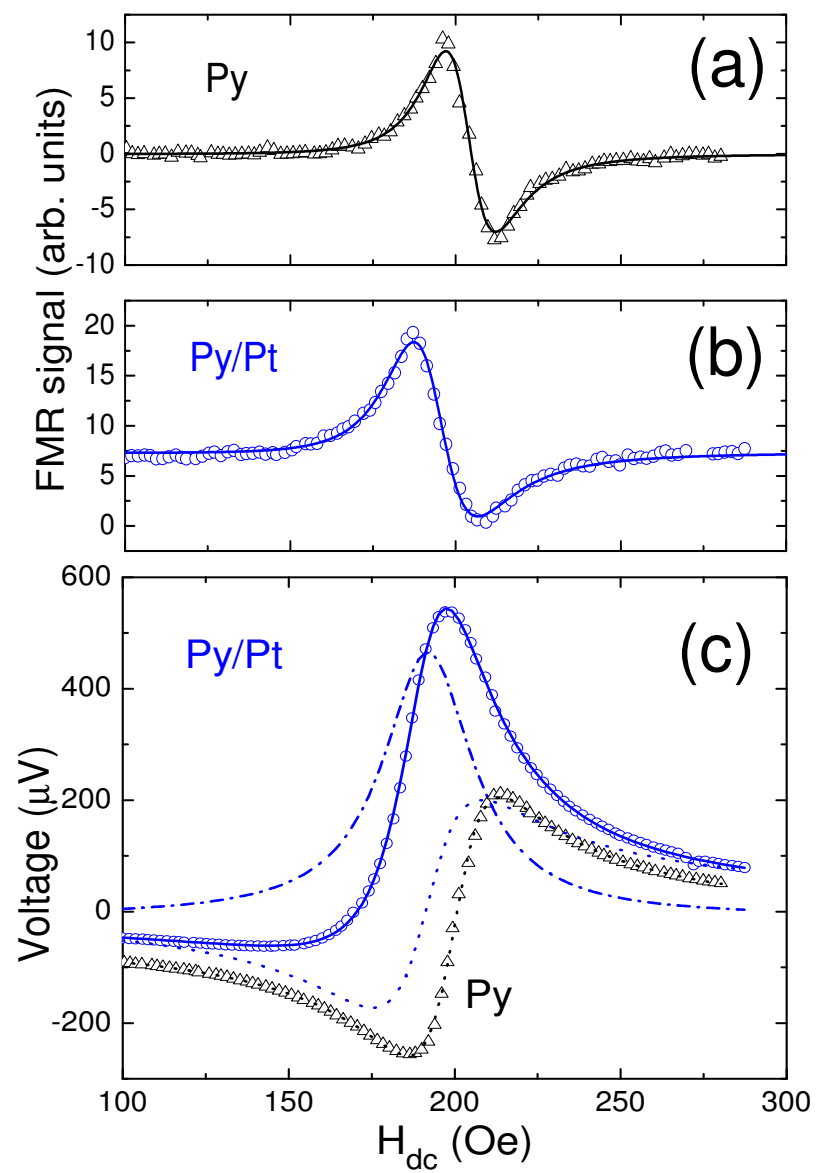

FIG. 2: (Color online) (a) and (b) FMR spectra (derivative) for $\mathrm{Py} \mid \mathrm{Pt}$ (blue $\circ$ ) and $\mathrm{Py}($ black $\triangle$ ). Solid lines are fits to a Lorentzian FMR absorption function. (c) Voltage along the samples vs. field $H_{d c}(\mathrm{Py} \mid \mathrm{Pt}$ : blue o; Py: black $\triangle)$. Dotted and dashed lines are fits to Eqs. (6) and (10), respectively; the solid line shows the combined fit for the $\mathrm{Py} \mid \mathrm{Pt}$ sample.

increase in the $d c$ voltage along the sample at the FMR position. However the lineshape is complicated: below the resonance field the voltage is negative, it changes sign just before the FMR resonance field, and has a positive tail in the high field region. In contrast, the single layer Py sample, which is not affected by spin pumping, shows a voltage signal that is purely antisymmetric with respect to the FMR position. The voltage due to ISHE depends only on the cone angle of the magnetization precession [see Eq. (2)] and thus must be symmetric with respect to the FMR resonance position. This means that the voltage measured in the $\mathrm{Py} \mid \mathrm{Pt}$ sample has two contributions: (i) a symmetric signal due to ISHE and (ii) an antisymmetric signal of the same origin as in the Py control sample.

The antisymmetric voltages observed in both $\mathrm{Py}$ and $\mathrm{Py} \mid \mathrm{Pt}$ originate from anisotropic magnetoresistance (AMR). Although the $\mathrm{MgO}$ provides $d c$ insulation be- 


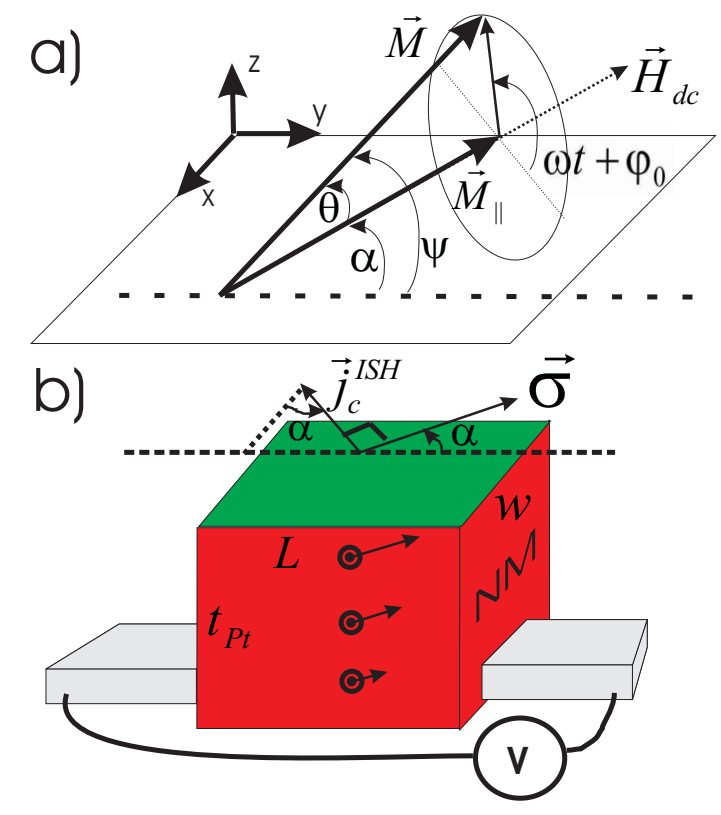

FIG. 3: (Color online) (a) Schematic of $\vec{M}$ precessing in Py. $\vec{M}$ precesses around its equilibrium direction given by $\vec{H}_{d c}$ at driving frequency $\omega$ and with phase delay $\varphi_{0}$ with respect to $h_{r f}$. $\alpha$ is the angle between $\vec{H}_{d c}$ and the waveguide axis (along $y$ ), $\theta$ is the cone angle described by $\vec{M}$ and $\psi$ is the angle between $\vec{M}$ and the waveguide axis. (b) Geometry of the $d c$ component of the pumped spin current with polarization direction $\vec{\sigma}$ along the equilibrium magnetization direction $\vec{M}_{||}$. The charge current due to ISHE $\vec{j}_{c}^{I S H}$ is orthogonal to the spin current direction (normal to the interface) and $\vec{\sigma}$. The voltage due to ISHE is measured along $y$ (waveguide axis). Solid arrows indicate the spin accumulation inside the $\mathrm{N}$, which decays with the spin diffusion length $\lambda_{s d}$.

tween the sample and the waveguide, there is strong capacitive coupling, and thus part of the $r f$ driving current $I_{r f}=I_{r f}^{m} \sin \omega t$ flows through the sample. This $r f$ current in the sample $I_{r f, S}$ flows along the waveguide direction and its magnitude can be estimated from the ratio between the waveguide resistance $R_{w g}$ and the sample resistance $R_{S}: I_{r f, S}=I_{r f} R_{w g} / R_{S}$. The precessing magnetization in the Py [see Fig. 3(a)] results in a time-dependent $R_{S}[\psi(t)]=R_{0}-\Delta R_{A M R} \sin ^{2} \psi(t)$ due to AMR given by $\Delta R_{A M R}$, where $R_{0}$ is the sample resistance with the magnetization along the waveguide axis and $\psi$ is the angle between the instantaneous magnetization $\vec{M}$ and the waveguide axis [see Fig. 3(a)] [26]. Since the AMR contribution to the resistance oscillates at the same frequency as the $r f$ current, a homodyne $d c$ voltage develops and is given by:

$$
V_{A M R}=I_{r f}^{m} \frac{R_{w g}}{R_{S}} \Delta R_{A M R} \frac{\sin (2 \theta)}{2} \frac{\sin (2 \alpha)}{2} \cos \varphi_{0},
$$

where $\varphi_{0}$ is the phase angle between magnetization precession and driving $r f$ field and the relation between $\theta$, $\alpha$ and $\psi$ is illustrated in Fig. 3(a). The phase angle $\varphi_{0}$ is zero well below the FMR resonance, $\pi / 2$ at the peak, and $\pi$ far above the resonance [27]. Thus $\cos \varphi_{0}$ changes sign upon going through the resonance and this gives rise to an antisymmetric $V_{A M R}$ as observed in both Py and $\mathrm{Py} \mid \mathrm{Pt}$ samples. Following Guan et al. [27] we calculate the cone angle $\theta$ and $\sin \varphi_{0}$ as a function of the applied field $H_{d c}$, FMR resonance field $H_{r}$, FMR linewidth $\Delta H$ and $r f$ driving field $h_{r f}$ :

$$
\begin{gathered}
\theta=\frac{h_{r f} \cos \alpha}{\Delta H \sqrt{1+\left(\frac{\left(H_{d c}-H_{r}\right)\left(H_{d c}+H_{r}+4 \pi M_{s}\right)}{\Delta H 4 \pi M_{s}}\right)^{2}}} \text {, and } \\
\sin \varphi_{0}=\frac{1}{\sqrt{1+\left(\frac{\left(H_{d c}-H_{r}\right)\left(H_{d c}+H_{r e s}+4 \pi M_{s}\right)}{\Delta H 4 \pi M_{s}}\right)^{2}}} .
\end{gathered}
$$

Using Eqs. (6-8) and taking a measured $0.95 \%$ value for $\Delta R_{A M R}$ fits the Py data [see Fig. 2(b)] with only one adjustable parameter $h_{r f}=4.5$ Oe.

In order to understand the $\mathrm{Py} \mid \mathrm{Pt}$ voltage data we have to include an additional contribution due to ISHE. In an open circuit an electric field $\vec{E}$ is generated leading to a total current density $\vec{j}(z)=\vec{j}_{c}^{I S H}(z)+\sigma_{N} \vec{E}$ with $\int \vec{j}(z) d z=0$ where $\sigma_{N}$ is the $\mathrm{N}$ conductivity. When the wire is much longer than thick, the electric field is constant in the wire and the component of the electric field along the measurement direction $y$ is:

$$
E_{y}=-\frac{\gamma}{\sigma_{N}} \frac{e \omega}{2 \pi} g_{\uparrow \downarrow} \sin \alpha \sin ^{2} \theta \frac{\lambda_{s d}}{t_{N}} \tanh \left(\frac{t_{N}}{2 \lambda_{s d}}\right) .
$$

Using Eq. 9 we calculate the voltage due to ISHE generated along the sample with length $L$ :

$$
V_{I S H}=-\frac{\gamma g_{\uparrow \downarrow} e L \lambda_{s d} \omega}{2 \pi \sigma_{N} t_{N}} \sin \alpha \sin ^{2} \theta \tanh \left(\frac{t_{N}}{2 \lambda_{s d}}\right) .
$$

Note that this voltage is proportional to $L$ and thus measurements of small $\gamma$ can be achieved by increasing the sample dimension. We used Eqs. (10) and (6) to fit the voltage measured for the Py|Pt sample, see solid line in Fig. 2(c). The dashed and dotted lines in Fig. 2(c) are the AMR and ISHE contributions, respectively. By using a literature value for $\mathrm{Pt}$ of $\lambda_{s d}=10 \pm 2 \mathrm{~nm}$ [28], the only remaining adjustable parameters are the $r f$ driving field $h_{r f}=4.5$ Oe and the spin Hall angle $\gamma=0.0067 \pm 0.0006$. Note that through the cone angle $\theta, h_{r f}$ enters both the AMR and ISHE contributions; this puts an additional constraint on this parameter, and, in fact, as seen from the fit to the control Py sample, it is already determined by the negative and positive tails of the AMR part.

This technique can be readily applied to determine $\gamma$

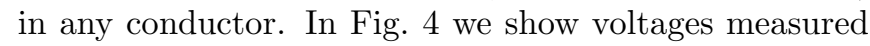
for $\mathrm{Py} \mid \mathrm{Au}$ and $\mathrm{Py} \mid \mathrm{Mo}$. The spin Hall contributions in $\mathrm{Au}$ and Mo are smaller than in $\mathrm{Pt}$, and note that for Mo the spin Hall contribution changes its sign. Fitting of the 

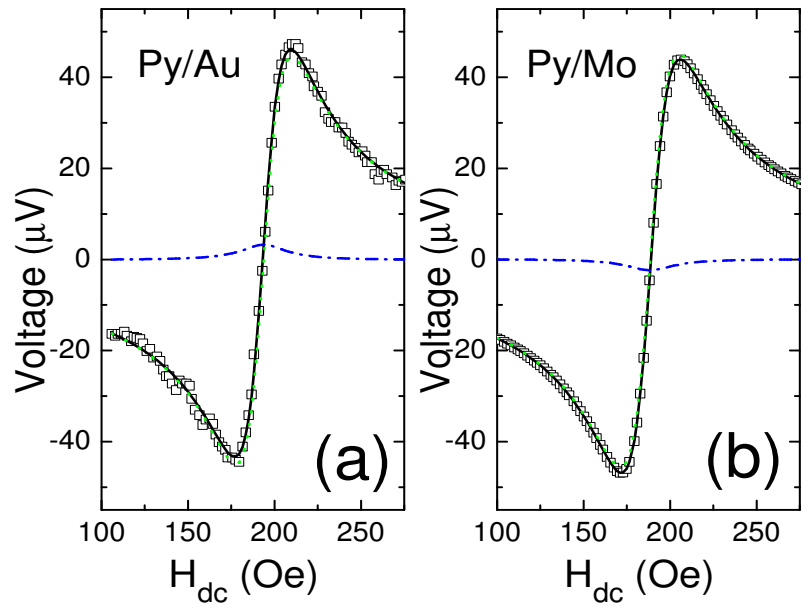

FIG. 4: (Color online) Voltage measured for (a) Py|Au and (b) $\mathrm{Py} \mid$ Mo. Shown are data (symbols), combined fits (solid lines) and individual AMR and ISHE contributions, with dotted (overlapping with solid) and dashed lines, respectiely. Note the opposite sign of ISHE contributions for Au and Mo.

TABLE I: Spin Hall angle $\gamma$ determined using $\lambda_{s d}$ and $\sigma_{N}$

\begin{tabular}{cccr}
\hline \hline Normal metal $\lambda_{s d}(\mathrm{~nm})$ & $\sigma_{N} 1 /(\Omega m)$ & \multicolumn{1}{c}{$\gamma$} \\
\hline $\mathrm{Pt}$ & $10 \pm 2$ & $(2.42 \pm 0.19) \times 10^{6}$ & $0.0067 \pm 0.0006$ \\
$\mathrm{Au}$ & $35 \pm 3$ & $(2.52 \pm 0.13) \times 10^{7}$ & $0.0016 \pm 0.0003$ \\
$\mathrm{Mo}$ & $35 \pm 3$ & $(4.66 \pm 0.23) \times 10^{6}$ & $-0.00023 \pm 0.00005$ \\
\hline \hline
\end{tabular}

data enabled us to extract the values of $\gamma$ for $\mathrm{Au}$ and Mo, see Table [. Note that the determination of $\gamma$ requires $\sigma_{N}$ and $\lambda_{s d}$ as an input parameters. $\sigma_{N}$ was measured using four-probe measurements for all samples. Reported values for $\lambda_{s d}$ vary considerably. We choose a conservatively low literature value for Pt from Ref. 28 and $\mathrm{Au}$ from Ref. 14, and for Mo we assumed that $\lambda_{s d}$ is comparable to Au. Even though this latter assumption may not necessarily hold, the sign change is consistent with earlier measurements [9]. Furthermore, our observed values for $\gamma$ are in good agreement with values reported by Otani et al. 9, 29] from measurements in lateral spin valves, but conflict with more optimistic values reported by other groups [8, 11]. We note that in lateral spin valves it is important to also understand the charge current contribution in order to rule out additional non-local voltage contributions [10]. In contrast, in our approach the spin pumping creates a uniform, macroscopic and well-defined spin current across the whole sample, and the voltage signal from spin Hall effects can readily be increased through use of longer samples, since $V_{I S H} \propto L$. Furthermore, using an integrated coplanar waveguide architecture provides control over parameters, such as the $r f$ driving field distribution. This enables us to carry out a quantitative analysis of the data, in contrast to the more qualitative description of the ISHE in Refs. 18 and 19 .

In conclusion, we performed FMR with simultaneous transverse voltage measurements in ferromagnetic/normal metal bilayers. From this we accurately determine the spin Hall angle for $\mathrm{Pt}, \mathrm{Au}$ and Mo by fitting the experimental data to a theory, which accounts for both the anistropic magnetoresistance and inverse spin Hall effect contributions. The combination of spin pumping and spin Hall effects provides a valuable technique for measuring spin Hall angle in many different materials.

We would like to thank R. Winkler, G. Mihajlović and M. Dyakonov for valuable discussions. This work was supported by U.S. DOE-BES under Contract No. DEAC02-06CH11357.

* Electronic address: mosendz@anl.gov

[1] M. Dyakonov and V. Perel, Sov. Phys. JETP Lett. 13, 467 (1971).

[2] J. Hirsch, Phys. Rev. Lett. 83, 1834 (1999).

[3] S. Zhang, Phys. Rev. Lett. 85, 393 (2000).

[4] M. I. Dyakonov and A. V. Khaetskii, in Spin Physics in Semiconductors, edited by M. I. Dyakonov (Springer, 2008), vol. 157 of Springer Series in Solid-State Sciences, chap. 8 , p. 212.

[5] A. Fert, A. Friederich, and A. Hamzic, J. Magn. Magn. Mater. 24, 231 (1981).

[6] S. O. Valenzuela and M. Tinkham, Nature 442, 176 (2006).

[7] T. Kimura, Y. Otani, T. Sato, S. Takahashi, and S. Maekawa, Phys. Rev. Lett. 98, 156601 (2007).

[8] T. Seki, Y. Hasegawa, S. Mitani, S. Takaashi, H. Imamura, S. Maekawa, J. Nitta, and K. Takanashi, Nature Mater. 7, 125 (2008).

[9] M. Morota, K. Ohnishi, T. Kimura, and Y. Otani, J. Appl. Phys. 105, 07C712 (2009).

[10] G. Mihajlović, J. E. Pearson, M. A. Garcia, S. D. Bader, and A. Hoffmann, Phys. Rev. Lett. 103, 166601 (2009).

[11] K. Ando, S. Takahashi, K. Harii, K. Sasage, J. Ieda, S. Maekawa, and E. Saitoh, Phys. Rev. Lett. 101, 036601 (2008).

[12] B. Heinrich, Y. Tserkovnyak, G. Woltersdorf, A. Brataas, R. Urban, and G. E. W. Bauer, Phys. Rev. Lett. 90, 187601 (2003).

[13] G. Woltersdorf, O. Mosendz, B. Heinrich, and C. H. Back, Phys. Rev. Lett. 99, 246603 (2007).

[14] O. Mosendz, G. Woltersdorf, B. Kardasz, B. Heinrich, and C. H. Back, Phys. Rev. B 79, 224412 (2009).

[15] Y. Tserkovnyak, A. Brataas, and G. E. W. Bauer, Phys. Rev. Lett. 88, 117601 (2002).

[16] Y. Tserkovnyak, A. Brataas, G. E. W. Bauer, and B. I. Halperin, Review of Modern Physics 77, 1375 (2005).

[17] circular precession was assumed for simplicity, realistic elliptical precession trajectory will result in a quantitative, but not qualitative modification to the model.

[18] E. Saitoh, M. Ueda, H. Miyajima, and G. Tatara, Appl. Phys. Lett 88, 182509 (2006).

[19] K. Ando, Y. Kajiwara, S. Takahashi, S. Maekawa, 
K. Takemoto, M. Takatsu, and E. Saitoh, Phys. Rev. B 78, 014413 (2008).

[20] A. Azevedo, L. H. V. Leao, R. L. Rodriguez-Suarez, A. B. Oliveira, and S. M. Rezende, J. Appl. Phys. 97, 10C715 (2005).

[21] O. Mosendz, B. Kardasz, and B. Heinrich, J. Appl. Phys. 103, 07B505 (2008).

[22] R. Urban, G. Woltersdorf, and B. Heinrich, Phys. Rev. Lett. 87, 217204 (2001).

[23] S. Mizukami, Y. Ando, and T. Miyazaki, J. Mag. Mag. Mat. 226, 1640 (2001).

[24] Y. Tserkovnyak, A. Brataas, and G. E. W. Bauer, Phys.
Rev. B 66, 224403 (2002).

[25] R. Cao, X. Fan, T. Moriyama, and J. Xiao, J. Appl. Phys. 105, $07 \mathrm{C} 705$ (2009).

[26] M. Costache, S. Watts, M. Sladkov, C. van der Wal, and B. van Wees, Appl. Phys. Lett 89, 232115 (2006).

[27] Y. Guan, W. E. Bailey, E. Vescovo, C. C. Kao, and D. A. Arena, J. Magn. Magn. Mat. 312, 374 (2006).

[28] H. Kurt, R. Loloee, K. Eid, J. W. P. Pratt, and J. Bass, Appl. Phys. Lett. 81, 4787 (2002).

[29] L. Vila, T. Kimura, and Y. Otani, Phys. Rev. Lett. 99, 226604 (2007). 